\title{
Effects of aerobic exercise, resistance exercise or both, on patient-reported health status and well-being in type 2 diabetes mellitus: a randomised trial
}

\author{
R. D. Reid • H. E. Tulloch • R. J. Sigal • G. P. Kenny • \\ M. Fortier • L. McDonnell • G. A. Wells • N. G. Boulé • \\ P. Phillips $\cdot$ D. Coyle
}

Received: 4 September 2009 /Accepted: 23 November 2009/Published online: 13 December 2009

(C) Springer-Verlag 2009

\begin{abstract}
Aims/hypothesis The Diabetes Aerobic and Resistance Exercise (DARE) study showed that aerobic and resistance exercise training each improved glycaemic control and that a combination of both was superior to either type alone in patients with type 2 diabetes mellitus. Here we report effects on patient-reported health status and well-being in the DARE Trial.

Methods We randomised 218 inactive participants with type 2 diabetes mellitus in parallel to 22 weeks of aerobic exercise $(n=51)$, resistance exercise $(n=58)$, combined aerobic and resistance exercise $(n=57)$ or no exercise (control; $n=52$ ). Intervention allocation was managed by a central office. Outcomes included health status as assessed by the physical and mental component scores of the Medical Outcomes Trust Short-Form 36-item version (SF36) and well-being as measured by the Well-Being
\end{abstract}

Electronic supplementary material The online version of this article (doi:10.1007/s00125-009-1631-1) contains supplementary material, which is available to authorised users.

R. D. Reid $(\bowtie) \cdot$ H. E. Tulloch $\cdot$ L. McDonnell $\cdot$ G. A. Wells University of Ottawa Heart Institute,

40 Ruskin Street,

Ottawa, ON, Canada K1Y 4W7

e-mail: breid@ottawaheart.ca

\section{R. J. Sigal}

Departments of Medicine, Cardiac Sciences and Community

Health Sciences, Faculties of Medicine and Kinesiology,

University of Calgary,

Calgary, AB, Canada

R. J. Sigal · G. P. Kenny • M. Fortier

School of Human Kinetics, Faculty of Health Sciences,

University of Ottawa,

Ottawa, ON, Canada
Questionnaire 12-item version (WBQ-12); these were measured at the Ottawa Hospital.

Results Using a $p$ value of 0.0125 for statistical significance due to multiple comparisons, mixed model analyses indicated that resistance exercise led to clinically but not statistically significant improvements in the SF-36 physical component score compared with aerobic exercise $(\Delta=2.7$ points; $p=0.048$ ) and control (i.e. no exercise; $\Delta=3.3$ points; $p=0.015)$. For mental component scores, there were clinically important improvements favouring no (control) compared with resistance $(\Delta=7.6$ points; $p<0.001)$ and combined $(\Delta=7.2$ points; $p<0.001)$ exercise. No effects on WBQ-12 scores were noted. Overall, 59/218 (27\%) of participants included in this analysis sustained an adverse event during the course of the study, including 16 participants in the combined exercise group, 19 participants in the resistance exercise group, 16 participants in the

N. G. Boulé

Faculty of Physical Education and Recreation,

University of Alberta,

Edmonton, AB, Canada

R. J. Sigal · P. Phillips

Clinical Epidemiology Program,

Ottawa Hospital Research Institute,

Ottawa, ON, Canada

D. Coyle

Department of Epidemiology and Community Medicine,

University of Ottawa,

Ottawa, ON, Canada 
aerobic exercise group, and eight participants in the control group. All participants were included in the intent-to-treat analyses. The trial is now closed to follow-up.

Conclusions/interpretation Resistance exercise was better than aerobic or no exercise for improving physical health status in these patients. No exercise was superior to resistance or combined exercise for improving mental health status. Well-being was unchanged by intervention.

\section{Trial registration: ClinicalTrials.gov NCT00195884}

Funding: This study was funded by the Canadian Institutes of Health Research (grant MCT-44155) and the Canadian Diabetes Association (The Lillian Hollefriend Grant).

Keywords Aerobic exercise $\cdot$ Diabetes mellitus $\cdot$ Health status · Quality of life · Resistance exercise $\cdot$ Well-being

$\begin{array}{ll}\text { Abbreviations } \\ \text { DARE } & \begin{array}{l}\text { Diabetes Aerobic and Resistance Exercise Trial } \\ \text { SF-36 }\end{array} \\ & \begin{array}{l}\text { Medical Outcomes Trust Short-Form 36-item } \\ \text { version }\end{array} \\ \text { WBQ-12 } & \text { Well-Being Questionnaire 12-item version }\end{array}$

\section{Introduction}

Regular exercise is a key part of the treatment plan for people with type 2 diabetes mellitus (along with diet, weight loss and in most cases insulin or medications) [1]. Meta-analyses and other studies have demonstrated that aerobic and resistance exercise each improves glycaemic control in people with this condition [2-7].

The Diabetes Aerobic and Resistance Exercise (DARE) Trial was a randomised controlled trial to determine the effects of aerobic exercise training, resistance exercise training and a combination of both in previously inactive, type 2 diabetic patients aged 39 to 70 years. Its primary results concerning effects on $\mathrm{HbA}_{1 \mathrm{c}}$ have been reported [8]. Both aerobic and resistance exercise training improved glycaemic control, while a combination of both was superior to either type of exercise training alone [8].

Health status and well-being are important outcomes, but have not been reported in most previous studies of exercise in people with type 2 diabetes mellitus. Several studies, however, have reported particularly beneficial effects of resistance exercise training on these outcomes in other clinical populations including patients with cardiac disease [9], the frail elderly [10] and men with prostate cancer receiving androgen deprivation therapy and/or radiotherapy $[11,12]$.

In the present paper, we report the effects of aerobic exercise training, resistance exercise training and a combination of both on pre-specified secondary outcomes related to health status and well-being in the DARE Trial. Our hypothesis was that participants randomised to either combined aerobic and resistance exercise training or resistance exercise training alone would show greater improvement in health status and well-being than those randomised to aerobic exercise training only or no exercise (waiting-list control). The rationale for our hypothesis was a previous study showing that strength training added to aerobic exercise led to additional incremental improvements in quality-of-life variables in patients with cardiac disease [9].

\section{Methods}

Design A complete description of the DARE study design and methods has been published [8]. This was a singlecentre, randomised controlled trial with a parallel group design. After a 4-week run-in period to assess compliance, previously inactive participants with type 2 diabetes mellitus were randomised to four groups: aerobic exercise training, resistance exercise training, combined aerobic and resistance exercise training or no exercise (waiting-list control). Health status and well-being were assessed at baseline, at the midpoint ( 3 months) and at the end of intervention ( 6 months). The study was approved by the Ottawa Hospital Research Ethics Board. All participants gave written informed consent.

Setting The 6-month exercise intervention took place at eight community-based exercise facilities in the OttawaGatineau region and was supervised by personal trainers.

Participants Previously inactive, type 2 diabetic patients aged 39 to 70 years were recruited through advertising, physicians and word-of-mouth. Inclusion criteria included type 2 diabetes mellitus as defined by the American Diabetes Association [13] for at least 6 months and baseline $\mathrm{HbA}_{1 \mathrm{c}}$ of $6.6 \%$ to $9.9 \%$. Potential participants were excluded if they: (1) were receiving insulin therapy; (2) were participating in exercise $\geq 2$ times per week for at least $20 \mathrm{~min}$ per session or had been in any resistance training during the previous 6 months; (3) reported changes in oral hypoglycaemic medications during the previous 2 months; (4) reported changes in antihypertensive or lipidlowering agents in the previous month; (5) reported a change of $\geq 5 \%$ in body weight during the previous 2 months; (6) had serum creatinine $\geq 200 \mu \mathrm{mol} / \mathrm{l}$; (7) had proteinuria $>1 \mathrm{~g} / 24 \mathrm{~h}$; (8) had blood pressure >160/95 mmHg; (9) had restrictions in physical activity due to disease; (10) had other medical conditions making participation inadvisable; or (11) completed fewer than ten of the 12 scheduled exercise sessions during the run-in period. 
Randomisation and blinded assessment Following completion of the run-in period, participants were randomly allocated in equal numbers to the aerobic, resistance, combined and no-exercise (control) groups. A central randomisation procedure with allocation concealment was used. The randomisation sequences were generated by an independent statistician using a software macro designed for this purpose. Randomisation was stratified by age (3954 or 55-70 years) and sex, with block sizes varying randomly between four and eight. The statistician provided the sequences directly to the Ottawa Hospital Research Institute Clinical Epidemiology Program Data Centre. DARE trial investigators and staff did not have access to the sequences at any point. It was not feasible to blind participants or intervention personnel to group assignment; however, assessment personnel were blinded.

Interventions Participants exercised three times per week, with training progressing gradually in length and intensity. The aerobic group exercised on treadmills and/or bicycle ergometers. Heart rate monitors (Polar Electro; Oy, Kempele, Finland) were used to adjust workloads to achieve target heart rate. Participants progressed from 15 to $20 \mathrm{~min}$ per session at $60 \%$ of maximum heart rate to 45 min per session at $75 \%$ of measured maximum heart rate. The resistance exercise group performed eight different exercises on weight machines each session, progressing to two to three sets of each exercise at a weight that could be lifted for a maximum of eight repetitions. The combined exercise group did the full aerobic programme plus the full resistance programme. A personal trainer was present for a minimum of three scheduled sessions weekly at each exercise facility and supervised all exercise programmes. After randomisation, he/she met each participant individually at least once a week for 4 weeks, every 2 weeks for the subsequent 2 months, then once a month for the remainder of the programme to ensure appropriate progression through the programme. The personal trainer monitored attendance and contacted the participant if any sessions were missed. After the initial 2 weeks post-randomisation, participants were free to attend at times most convenient for them, but they were required to come at least once a week at a time when the personal trainer was present. Control participants were asked to revert to their pre-study activity levels. Efforts were made to minimise dietary and medication co-intervention.

Outcomes Health status and well-being were measured at baseline, 3 months (midpoint) and 6 months (end of intervention) using the Medical Outcomes Trust ShortForm 36-item version (SF-36) [14] questionnaires and a slightly modified version of the Well-Being Questionnaire 12-item version (WBQ-12) [15, 16], respectively.
The SF-36 is a 36 -item, self-administered, health status assessment that yields physical and mental health component scores. The physical component summary and mental component summary scales are scored with weights and normalised to a population mean of 50 and SD of 10 . In diabetic populations, 2.0 to about 2.5 points is a reasonable estimate of the smallest clinically important difference in either physical or mental component summary scores [17]. The validity of the SF-36 has been demonstrated in several studies of diabetic participants [14, 18-22].

The WBQ-12 is a reliable and valid measure of wellbeing for patients with type 2 diabetes mellitus $[15,16]$. The General Well-Being score is the sum of all 12 items and is scored from 0 to 36 , where a higher score indicates a greater level of well-being; it was used as the primary indicator of well-being in the present study. The minimal clinically important difference in the WBQ-12 has not yet been determined [23]. The WBQ-12 used was slightly modified; on five items, the wording was changed from 'I feel...' to 'I have felt...' We conducted a factor analysis and confirmed the factor structure of the modified WBQ- 12 . High Cronbach's $\alpha$ coefficients demonstrated good internal consistency reliability in the entire scale $(\alpha=0.87)$.

Statistical analysis The number of participants in the original DARE study with complete baseline health status and well-being data determined the sample size in the present study. Of the 251 participants in our trial, 33 did not complete the baseline health status questionnaires and were therefore excluded. Baseline comparisons were performed using univariate ANOVA for continuous variables and $\chi^{2}$ analyses for categorical variables. Mixed-model repeated measures analysis was used to compare the differences between groups over time. In the repeated measures factorial design with two factors, the within-participants factor was time (baseline, mid-point and end of intervention) and the between-participants factor was group (resistance, aerobic, combined exercise, control). Our primary analysis considered health status or well-being by time, group and group $\times$ time interaction, but we also tested a model that adjusted for age, sex, baseline $\mathrm{HbA}_{1 \mathrm{c}}$ and BMI. Mixed-model repeated measures analysis is based on likelihood methods and was conducted, in part, because of its flexibility in dealing with missing data. The advantage of this form of analysis is that it retains all participants who have provided at least one data point [24]. For all analyses, an intention-to-treat approach was followed. We also examined Pearson correlations for changes in health status and well-being and for changes in $\mathrm{HbA}_{1 \mathrm{c}}$, aerobic fitness, muscular fitness and body composition. For exploratory purposes, we conducted secondary analyses to determine if adverse events influenced the results for health status or well-being. Alpha was set at 0.0125 for inter-group 
comparisons to adjust for four pre-specified comparisons: resistance exercise vs control, combined exercise vs control, resistance vs aerobic exercise and combined vs aerobic exercise. All analyses were done using SAS Version 9.1 (SAS Institute, Cary, NC, USA).

\section{Results}

Between October 1999 and December 2003, 2,282 people were screened. Figure 1 shows the flow of participants from recruitment to follow-up. As shown by $\chi^{2}$ analyses and $t$ tests, individuals who did not complete the health status and well-being measures at baseline $(n=33)$ were not significantly different from those who completed the questionnaires on a number of variables including: age (53.6 vs 54.2 years old; $p=0.67)$, sex ( $48 \%$ vs $65 \%$ male; $p=0.06$ ), time since diagnosis ( 4.8 vs 5.4 years; $p=0.57$ ) and both baseline BMI (33.7 vs $\left.33.5 \mathrm{~kg} / \mathrm{m}^{2} ; p=0.82\right)$ and $\mathrm{HbA}_{1 \mathrm{c}}(7.7 \%$ vs $7.7 \% ; p=0.69)$.

Table 1 shows the participants' baseline characteristics. The groups were comparable in age, sex distribution, duration of diabetes and BMI. Median exercise training attendance (as determined by a review of exercise log sheets) was $86 \%$ (interquartile range $74-92 \%$ ) in the combined exercise group, $80 \%$ (interquartile range 46$93 \%$ ) in the aerobic exercise group and $85 \%$ (interquartile range $72-91 \%$ ) in the resistance exercise group. One person assigned to aerobic exercise reported participating in resistance training; no one assigned to resistance exercise, combined exercise or control groups reported exercise training outside their assigned DARE group. Overall, $59 / 218(27 \%)$ of participants included in this analysis sustained an adverse event during the course of the study, including 16 participants in the combined exercise group, 19 participants in the resistance exercise group, 16 participants in the aerobic exercise group, and eight participants in the control group. The main outcomes of the trial have been reported previously [8]. Briefly, the absolute change in the $\mathrm{HbA}_{1 \mathrm{c}}$ value in combined compared with control groups was -0.96 percentage points $(95 \% \mathrm{CI}$ $-1.32,-0.61)$. The change in $\mathrm{HbA}_{1 \mathrm{c}}$ in resistance exercise compared with control groups was -0.37 percentage points (95\% CI $-0.73,-0.02)$. The change in combined compared with aerobic groups was -0.46 percentage points $(95 \% \mathrm{CI}$ $-0.83,-0.90)$ and the change in resistance compared with aerobic groups was 0.13 percentage points $(95 \% \mathrm{CI}-0.24$, $0.50)$.

Changes in health status Changes in health status as measured by the physical and mental component summary scores of the SF-36 are shown in Table 2. The analysis for physical component summary showed no main effects for group $(F=0.37 ; p=0.774)$ or time $(F=1.84 ; p=0.161)$, but a

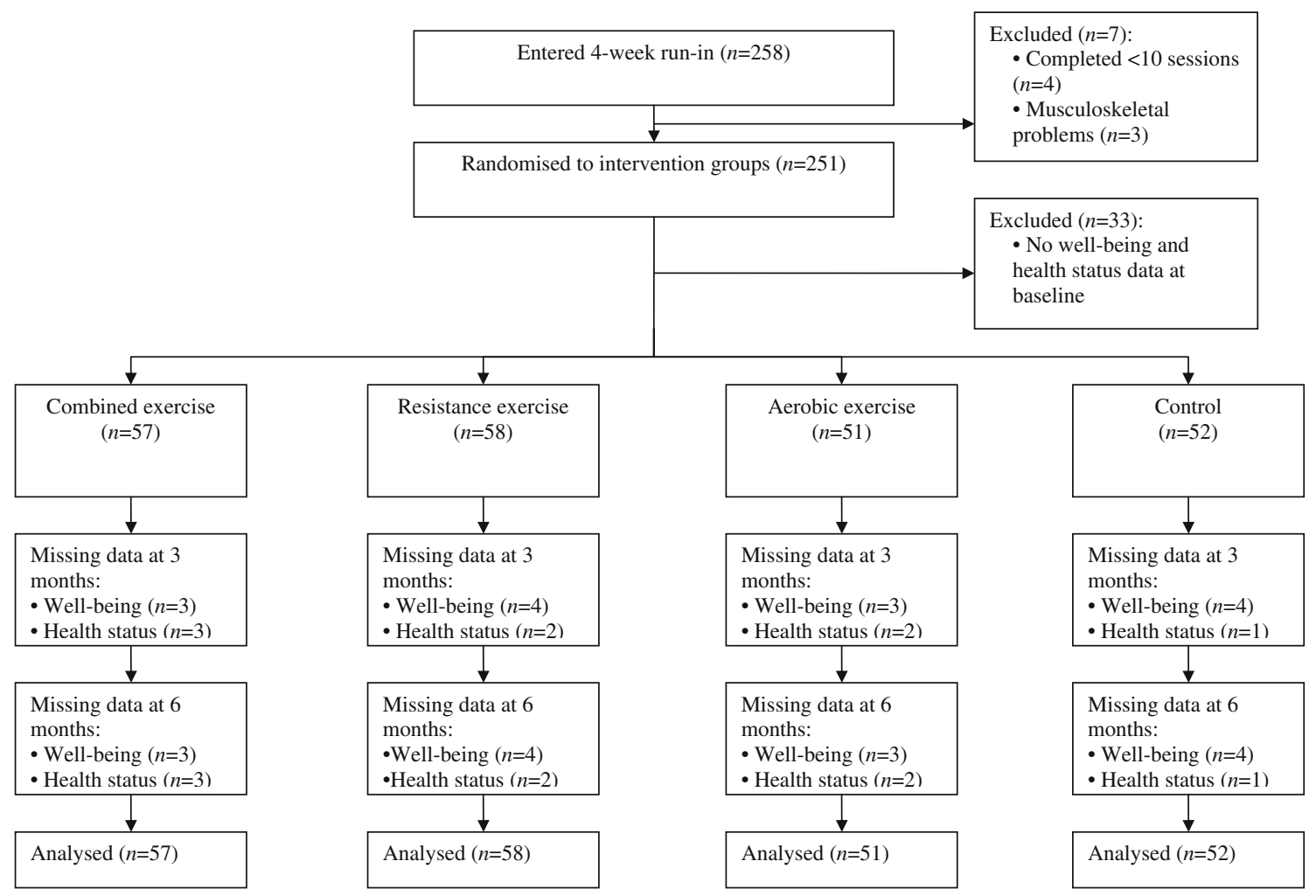

Fig. 1 Participant flow through allocation and follow-up 
Table 1 Baseline characteristics of study participants

\begin{tabular}{|c|c|c|c|c|c|}
\hline \multirow[t]{2}{*}{ Variables } & \multicolumn{5}{|c|}{ Exercise groups } \\
\hline & $\begin{array}{l}\text { Overall } \\
(n=218)\end{array}$ & $\begin{array}{l}\text { Combined } \\
(n=57)\end{array}$ & $\begin{array}{l}\text { Resistance } \\
(n=58)\end{array}$ & $\begin{array}{l}\text { Aerobic } \\
(n=51)\end{array}$ & $\begin{array}{l}\text { Control } \\
(n=52)\end{array}$ \\
\hline \multicolumn{6}{|l|}{ Demographic characteristics } \\
\hline Age (years) & $54.2(7.1)$ & $53.3(7.2)$ & $54.7(7.6)$ & $53.8(6.4)$ & $55.2(6.9)$ \\
\hline Men/women $(n / n)$ & $142 / 76$ & $38 / 19$ & $38 / 20$ & $33 / 18$ & $33 / 19$ \\
\hline \multicolumn{6}{|l|}{ Clinical characteristics } \\
\hline Duration of diabetes (years) & $5.4(4.4)$ & $5.2(4.8)$ & $6.1(4.7)$ & $5.1(3.5)$ & $5.0(4.5)$ \\
\hline $\mathrm{HbA}_{1 \mathrm{c}}(\%)$ & $7.7(0.9)$ & $7.7(0.9)$ & $7.7(0.9)$ & $7.7(0.9)$ & $7.7(0.9)$ \\
\hline BMI $\left(\mathrm{kg} / \mathrm{m}^{2}\right)$ & $33.5(5.8)$ & $33.7(6.1)$ & $32.9(5.6)$ & $34.3(5.9)$ & $33.2(5.5)$ \\
\hline \multicolumn{6}{|l|}{ Health status and well-being } \\
\hline SF-36 physical component summary score & $49.2(7.4)$ & $49.4(7.4)$ & $48.3(7.5)$ & $49.7(7.2)$ & $49.2(7.4)$ \\
\hline SF-36 mental component summary score & $51.0(9.5)$ & $52.9(9.5)$ & $51.2(9.6)$ & $52.4(9.3)$ & $47.3(9.6)^{\mathrm{a}}$ \\
\hline WBQ-12 general well-being score & $26.1(5.4)$ & $27.7(5.4)$ & $26.6(5.7)$ & $25.6(5.2)$ & $24.6(5.3)$ \\
\hline
\end{tabular}

Unless otherwise indicated, values are mean (SD)

The SF-36 physical and mental component summary scales were transformed to allow norm-based scoring. Anytime a physical and mental component summary score was less than 50 , health status was below average; conversely anytime a score was above 50 , health status was above average. The 'expected' range (i.e. mean $\pm 3 \mathrm{SD}$ ) was 20 to 58 for the physical component summary and 17 to 62 for the mental component summary

The general well-being score of the WBQ-12 is the sum of all 12 items and is scored from 0 to 36, where a higher score indicates a greater level of well-being

${ }^{a}$ SF-36 mental component summary score significantly lower in Control group than in combined, resistance or aerobic exercise groups at baseline

significant interaction for group and time $(F=2.32$; $p=0.03$ ). Planned contrasts showed that there were clinically important improvements in the physical component summary score favouring the resistance compared with the aerobic $(\Delta=2.7$ points; $p=0.048)$ and control $(\Delta=3.3$ points; $p=0.015)$ groups. However, these differences were not statistically significant after adjustment for multiple comparisons (i.e. $p<0.0125$ ). The analysis of mental component summary scores showed no main effect for group $(F=1.16 ; p=0.092)$, but a significant main effect of time $(F=8.63 ; p<0.001)$ and a significant interaction for group and time $(F=4.39 ; p<0.001)$. There were clinically important improvements favouring control compared with resistance $(\Delta=7.6$ points; $p<0.001)$ and combined $(\Delta=7.2$ points; $p<0.001)$ groups. Results were unchanged after adjustment for age, sex, baseline $\mathrm{HbA}_{1 \mathrm{c}}$ and $\mathrm{BMI}$.

Changes in well-being Changes in general well-being measured by the WBQ-12 are shown in Table 2. The analysis of general well-being showed a significant main effect for group $(F=3.17 ; p=0.025)$ and a significant main effect towards improved well-being for time $(F=11.62$; $p<0.001)$. The interaction between group and time was not significant $(F=0.31 ; p=0.930)$. Planned contrasts showed no between-group differences for general well-being from baseline to 6 months. Results were unchanged after adjustment for age, sex, baseline $\mathrm{HbA}_{1 \mathrm{c}}$ and BMI.
Associations between changes in well-being, health status, $H b A_{1 c}$, aerobic fitness, muscular fitness and body composition For exploratory purposes, we examined Pearson correlations for changes in health status, well-being, $\mathrm{HbA}_{1 \mathrm{c}}$, aerobic fitness, muscular fitness and body composition over the intervention period (Electronic supplementary material [ESM] Table 1). Changes in health status and well-being were unrelated to changes in $\mathrm{HbA}_{1 \mathrm{c}}$, aerobic fitness, muscular fitness or body composition.

Associations between adverse events and changes in health status or well-being For exploratory purposes, we re-ran the mixed model including a dichotomous term for the occurrence of any adverse event, and a term for the interaction between the occurrence of adverse event and time. The adverse event $\times$ time $\times$ group interactions for physical component summary score $(p=0.111)$, mental component summary score $(p=0.927)$, and well-being ( $p=0.767)$ were not significant, indicating that the overall results were not influenced by the adverse event status.

\section{Discussion}

We compared combined aerobic and resistance exercise training and resistance exercise alone to aerobic exercise training and a non-exercising control condition for their 
Table 2 Changes in health status and well-being

\begin{tabular}{|c|c|c|c|c|c|}
\hline Variables per group & Baseline & 3 months & 6 months & Difference in change from baseline to 6 months & $p$ value \\
\hline \multicolumn{6}{|c|}{ SF-36 physical component scale } \\
\hline Combined $(n=57)$ & $49.4(7.4)$ & $49.3(7.4)$ & $49.7(7.5)$ & & \\
\hline Resistance $(n=58)$ & $48.3(7.5)$ & $51.6(7.5)$ & $51.2(7.5)$ & & \\
\hline Aerobic $(n=51)$ & $49.7(7.2)$ & $49.6(7.5)$ & $49.9(7.5)$ & & \\
\hline Control $(n=52)$ & $49.2(7.4)$ & $49.2(7.4)$ & $48.8(7.5)$ & & \\
\hline \multicolumn{6}{|l|}{ Intergroup comparisons } \\
\hline Combined vs control & & & & $0.6(-2.0,3.3)$ & 0.632 \\
\hline Resistance vs control & & & & $3.3(1.0,5.5)$ & 0.015 \\
\hline Combined vs aerobic & & & & $-0.1(-2.8,2.6)$ & 0.939 \\
\hline Resistance vs aerobic & & & & $2.7(0.1,5.2)$ & 0.048 \\
\hline \multicolumn{6}{|c|}{ SF-36 mental component scale } \\
\hline Combined $(n=57)$ & $52.9(9.5)$ & $53.5(9.5)$ & $52.4(9.6)$ & & \\
\hline Resistance $(n=58)$ & $51.2(9.6)$ & $51.7(9.6)$ & $50.4(9.6)$ & & \\
\hline Aerobic $(n=51)$ & $52.4(9.3)$ & $54.5(9.6)$ & $54.5(9.6)$ & & \\
\hline Control $(n=52)$ & $47.3(9.6)$ & $53.1(9.5)$ & $54.0(9.6)$ & & \\
\hline \multicolumn{6}{|l|}{ Intergroup comparisons } \\
\hline Combined vs control & & & & $-7.2(-10.7,-3.8)$ & $<0.001$ \\
\hline Resistance vs control & & & & $-7.6(-11.0,-4.2)$ & $<0.001$ \\
\hline Combined vs aerobic & & & & $-2.7(-6.2,0.9)$ & 0.139 \\
\hline Resistance vs aerobic & & & & $-3.0(-6.5,0.5)$ & 0.089 \\
\hline \multicolumn{6}{|c|}{ WBQ-12 general well-being } \\
\hline Combined $(n=57)$ & $27.7(5.4)$ & $28.6(5.4)$ & $28.6(5.4)$ & & \\
\hline Resistance $(n=58)$ & $26.6(5.7)$ & $27.4(5.6)$ & $27.6(5.6)$ & & \\
\hline Aerobic $(n=51)$ & $25.6(5.2)$ & $27.3(5.3)$ & $27.3(5.3)$ & & \\
\hline Control $(n=52)$ & $24.6(5.3)$ & $25.6(5.3)$ & $25.9(5.4)$ & & \\
\hline \multicolumn{6}{|l|}{ Intergroup comparisons } \\
\hline Combined vs control & & & & $-0.4(-2.0,1.1)$ & 0.578 \\
\hline Resistance vs control & & & & $-0.3(-1.9,1.3)$ & 0.689 \\
\hline Combined vs aerobic & & & & $-0.8(-2.4,0.8)$ & 0.326 \\
\hline Resistance vs aerobic & & & & $-0.7(-2.3,0.9)$ & 0.326 \\
\hline
\end{tabular}

Values for baseline, 3 months and 6 months are mean (SD). Values for difference in change from baseline to 6 months are mean (95\% CI).

The SF-36 physical and mental component summary scales were transformed to allow norm-based scoring. Anytime a physical and mental component summary score was less than 50 , health status was below average; conversely anytime a score was above 50 , health status was above average. The 'expected' range (i.e. mean $\pm 3 \mathrm{SD}$ ) was 20 to 58 for the physical component summary and 17 to 62 for the mental component summary

The general well-being score of the WBQ-12 is the sum of all 12 items and is scored from 0 to 36, where a higher score indicates a greater level of well-being

effects on health status and well-being in patients with type 2 diabetes mellitus. Resistance exercise led to clinically but not statistically significant improvements in physical health status. Surprisingly, no-exercise control participants improved their mental health status more than those doing combined aerobic and resistance or resistance alone exercise. Well-being, as measured by the WBQ-12, was unchanged by the exercise interventions. Our hypothesis that participants randomised to either combined aerobic and resistance exercise training or resistance exercise alone would show greater improvement in well-being and health status than those randomised to aerobic exercise training or waiting-list control was only partially supported.

The current study has methodological strengths and limitations. Strengths include the randomised design, high adherence to the exercise regimens and the concurrent measurement of biological, health status and well-being outcomes. The main limitations include our inability to blind participants to treatment group assignment, statistical power for some outcomes, the generic nature of the SF-36 instrument and the multiplicity of interventions, outcomes and analyses. Blinding of participants to group assignment 
is not feasible in most clinical trials employing behavioural interventions. In randomised trials of exercise interventions, exercise contamination can occur when the control group engages in significant exercise during the intervention period or when participants assigned to one form of exercise (i.e., resistance or aerobic) engage in the other form [25]. Exercise contamination would tend to reduce observed differences between groups. We do not believe exercise contamination was a problem in the present study. Only one person assigned to aerobic training reported participating in resistance training; no one assigned to resistance or combined aerobic and resistance exercise training or the control group reported engaging in aerobic or resistance activity beyond pre-study levels. Additionally, outside of the DARE exercise sessions, background physical activity recorded with pedometers did not change materially over time in any group. It is unlikely that statistical power limited our ability to detect clinically important difference in the WBQ-12. The 95\% CI around the point estimates of the between-group differences for change in WBQ-12 indicate that the likely effect of exercise is at most a difference of about two points (i.e. small and of little clinical significance). On the other hand, the $95 \%$ CIs in Table 2 suggest we may have had insufficient power to detect small but potentially important differences of about two to five points between groups in the physical and mental components of the SF-36. Our sample size was limited to the number of participants in the original DARE study with complete baseline health status and well-being data. The SF-36 is a widely used generic measure of health status with very good psychometric properties [26]. Nonetheless, it is not clear whether it taps into all relevant aspects of the experiences of people living with diabetes. The SF-36 may be relatively unresponsive to important benefits and consequences of different types of exercise training in this population. It is possible that a measure more specific to the issues of people with diabetes would have been more responsive to the effects of the different training regimens. Since we had four inter-group comparisons, the alpha for significance was set at 0.0125 ; this may be why some clinically important differences did not reach the threshold for statistical significance.

The present study adds significantly to the literature on the health benefits of exercise for type 2 diabetes mellitus patients. Ligtenberg [27] examined the effects of supervised aerobic exercise training in 51 type 2 diabetic patients and found improvements in aerobic capacity and blood lipid variables that favoured the exercise training group compared with a control group; there were no improvements in glycaemic control or insulin sensitivity. In a subsequent analysis [28], they found improvements in anxiety, energy and positive well-being, but not depression (measured by the 22-item version of the WBQ) favouring the aerobic exercise training group. Using a larger sample size, a longer training period and interventions based on aerobic and resistance exercise, our study was unable to replicate these findings for well-being. Kirk [29] evaluated the effect of exercise consultation on promotion of physical activity in people with type 2 diabetes. In that study, 26 sedentary people with type 2 diabetes were randomly assigned to receive an exercise consultation and standard exercise information (experimental) or standard exercise information alone (control). Participants receiving an exercise consultation increased their stage of exercise behaviour compared with controls and a significant difference was recorded for the change in accelerometer-measured activity counts per week from baseline to follow-up between the experimental and control groups. The vitality and mental health scales of the SF-36 improved in the experimental group from baseline to follow-up at 5 weeks, but only the mental health scale showed significant between-group differences for change in score from baseline to follow-up. Well-being assessed by the 22-item WBQ trended toward positive changes in the experimental group compared with control; however, none of these changes were statistically significant. Unlike the Kirk study, the present study failed to demonstrate improvements in the mental component of health status with a longer exercise intervention relative to control. We did, however, observe benefits of resistance exercise training for the physical component. In another randomised controlled trial in 279 post-menopausal women with type 2 diabetes mellitus [30], Toobert found that after 6 months participation in a self-directed physical activity programme including moderate aerobic activity for $30 \mathrm{~min}$ most days of the week and ten strength-training exercises performed twice per week improvements for $\mathrm{HbA}_{1 \mathrm{c}}$, BMI and plasma fatty acids were significantly greater in the intervention group than in the usual care group. There were no significant between-group (experimental vs usual care) differences in the physical and mental health scores of the MOS-12, a brief version of the SF-36. The present study found benefits for the physical component of the SF-36 for supervised resistance exercise training performed at weights that could be lifted for a maximum of eight repetitions, three times per week in men and women with type 2 diabetes. Martin et al. found aerobic exercise-induced improvements in health status were dose-dependent in a randomised controlled trial conducted in 430 sedentary postmenopausal women with elevated systolic blood pressure [31]. In contrast, the present study suggests that health status improvements in patients with type 2 diabetes are not dose-dependent; combined aerobic and resistance exercise training was inferior to resistance exercise training alone with regard to enhancing the physical component of health status. Exploratory analyses suggest that changes in well-being and health status were 
unrelated to changes in $\mathrm{HbA}_{1 \mathrm{c}}$, aerobic fitness, muscular fitness or body composition.

There are at least two possible explanations for our observation that resistance exercise training improved the physical component of health status, while combined aerobic and resistance exercise training did not. First, as discussed above, the 95\% CIs in Table 2 suggest we may have had insufficient power to detect small but potentially important differences of about two to three points between groups in the physical component of the SF-36. Second, combined aerobic and resistance exercise training may have left people more fatigued than resistance exercise training alone. Participants assigned to the combined exercise group completed work-outs that were almost twice as long as participants assigned to resistance or aerobic exercise alone; these participants may have felt that the training required too much time and effort, and interfered with other important things in their life.

Changes in the mental component of the SF-36 favoured the control group compared with any of the exercise intervention groups; however, between-group differences were the result of a moderately large improvement in the mental component summary scores for the control group, rather than any detrimental effect of the exercise interventions on these scores. We believe that the observed changes in the mental component summary scores reflect the statistical phenomenon of reversion to the mean. Reversion to the mean refers to a situation where a variable that is extreme on its first measurement will tend to be closer to the centre of the distribution at a later measurement. Thus lower scores on the pre-test will tend to be higher on the post-test and higher scores on the pre-test will tend to lower on the post-test. Importantly, reversion is always toward the population mean of the group. Despite random assignment, the mean score for the mental component summary in the control group was significantly lower at the pre-test than the mean scores for the combined, resistance and aerobic exercise groups. Mean scores in the combined, resistance and aerobic exercise groups were close to the expected population mean of 50, while the mean score in the control group was much lower than expected. At the post-test, the mean mental component summary scores in the combined and resistance exercise groups remained relatively stable compared with the pre-test, while mental component summary scores in the aerobic exercise group showed a small improvement. Over the same time, the mean mental component summary score in the control group increased back toward the expected population mean.

These results apply to type 2 diabetes mellitus patients who are generally well-managed and motivated to participate in a structured and supervised exercise programme. The results reported here reflect changes in health status and well-being during the trial; they do not reflect any potential future effects on health status and well-being related to long-term effects of improved glycaemic control on complications of diabetes. Participants in this study had mean SF-36 physical and mental component summary scores of 49.2 (SD 7.4) and 51.0 (SD 9.5) at baseline, respectively. This suggests that they had little meaningful impairment of health status compared with Canadians without type 2 diabetes [32]. It is possible that the exercise interventions could have lead to greater changes in health status and well-being in type 2 diabetes mellitus patients with greater relative impairment in these areas. Future studies are needed to explore the benefits of different exercise training programmes (including types, duration, different level of supervision, different support programmes) and to determine whether these effects can also be achieved in diabetic patients who are already active or in those with insulin-dependent diabetes.

To date, most studies of exercise in patients with type 2 diabetes mellitus have suggested that exercise has positive effects on well-being and physical and mental aspects of health status. However, these studies have used noncontrolled designs, enrolled small numbers of patients and have not considered the effects of resistance exercise training. In this randomised controlled trial, aerobic exercise training, resistance exercise training and a combination of both failed to improve patient-reported wellbeing. Resistance exercise training caused some clinically important improvements in physical health status; however, none of the exercise training programmes enhanced the mental component of health status relative to non-exercise control. While aerobic and resistance exercise training and a combination of both unmistakably improve glycaemic control [2,8], effects on patient-reported quality of life outcomes during a 6-month exercise intervention are less clear.

Acknowledgements The authors thank the DARE study participants. We also thank D. Pepin, K. Fetch, R. Attema, K. Dittmann, K. Phillips, P. Healey, K. Holland, J. Murrin, N. McInnis, J. Fetch and T. Leech, students in the School of Human Kinetics, University of Ottawa, and the Ottawa-Carleton Regional YMCA/YWCA and Nautilus Plus of Gatineau, Québec, Canada, for their contributions to study coordination, exercise training and evaluation of study participants. We thank C. Bradley for allowing us to use her WellBeing Questionnaire and for her helpful comments on a draft of this manuscript. We also thank J. Jaffey and F. Khandwala for their assistance with data analysis. The DARE trial was supported by grants from the Canadian Institutes of Health Research (grant MCT-44155) and the Canadian Diabetes Association (The Lillian Hollefriend Grant). R. D. Reid was supported by a New Investigator Award from the Heart and Stroke Foundation of Canada. H. E. Tulloch was supported by a Doctoral Research Award from the Social Sciences and Humanities Research Council of Canada. R. J. Sigal was supported by a New Investigator Award from the Canadian Institutes of Health Research and the Ottawa Health Research Institute Lifestyle Research Chair, and by a Senior Health Scholar Award from the Alberta Heritage Foundation for Medical Research. G. P. Kenny was 
supported by a Career Scientist Award from the Ontario Ministry of Health and Long Term Care and a University of Ottawa Research Chair. N. G. Boulé was supported by a Postgraduate Scholarship from the National Sciences and Engineering Research Council of Canada.

Duality of interest The authors declare that there is no duality of interest associated with this manuscript.

\section{References}

1. Sigal RJ, Kenny GP, Wasserman DH, Castaneda-Sceppa C, White RD (2006) Physical activity/exercise and type 2 diabetes: a consensus statement from the American Diabetes Association. Diabetes Care 29:1433-1438

2. Boule NG, Haddad E, Kenny GP, Wells GA, Sigal RJ (2001) Effects of exercise on glycemic control and body mass in type 2 diabetes mellitus: a meta-analysis of controlled clinical trials. JAMA 286:1218-1227

3. Castaneda C, Layne JE, Munoz-Orians L et al (2002) A randomized controlled trial of resistance exercise training to improve glycemic control in older adults with type 2 diabetes. Diabetes Care 25:2335-2341

4. Dunstan DW, Daly RM, Owen N et al (2002) High-intensity resistance training improves glycemic control in older patients with type 2 diabetes. Diabetes Care 25:1729-1736

5. Eves ND, Plotnikoff RC (2006) Resistance training and type 2 diabetes: considerations for implementation at the population level. Diabetes Care 29:1933-1941

6. Snowling NJ, Hopkins WG (2006) Effects of different modes of exercise training on glucose control and risk factors for complications in type 2 diabetic patients: a meta-analysis. Diabetes Care 29:2518-2527

7. Thomas DE, Elliott EJ, Naughton GA (2006) Exercise for type 2 diabetes mellitus. Cochrane Database Syst Rev, Issue 3. Art no.: CD002968. doi:10.1002/14651858.CD002968.pub2

8. Sigal RJ, Kenny GP, Boule NG et al (2007) Effects of aerobic training, resistance training, or both on glycemic control in type 2 diabetes: a randomized trial. Ann Intern Med 147:357-369

9. Beniamini Y, Rubenstein JJ, Zaichkowsky LD, Crim MC (1997) Effects of high-intensity strength training on quality-of-life parameters in cardiac rehabilitation patients. Am J Cardiol 80:841-846

10. Fiatarone MA, O’Neill EF, Ryan ND et al (1994) Exercise training and nutritional supplementation for physical frailty in very elderly people. N Engl J Med 330:1769-1775

11. Segal RJ, Reid RD, Courneya KS et al (2003) Resistance exercise in men receiving androgen deprivation therapy for prostate cancer. J Clin Oncol 21:1653-1659

12. Segal RJ, Reid RD, Courneya KS et al (2009) Randomized controlled trial of resistance or aerobic exercise in men receiving radiation therapy for prostate cancer. J Clin Oncol 27:344-351

13. No authors listed (1997) Report of the Expert Committee on the diagnosis and classification of diabetes mellitus. Diabetes Care 20:1183-1197

14. Ware JE, Snow KK, Kosinski M, Gandek B (1993) SF-36 health survey: manual and interpretation guide. Nimrod, Boston
15. Lewis KS, Jennings AM, Ward JD, Bradley C (1990) Health belief scales developed specifically for people with tablet-treated type 2 diabetes. Diabet Med 7:148-155

16. Pouwer F, van der Ploeg HM, Ader HJ, Heine RJ, Snoek FJ (1999) The 12-item well-being questionnaire. An evaluation of its validity and reliability in Dutch people with diabetes. Diabetes Care 22:2004-2010

17. Samsa G, Edelman D, Rothman ML, Williams GR, Lipscomb J, Matchar D (1999) Determining clinically important differences in health status measures: a general approach with illustration to the Health Utilities Index Mark II. Pharmacoeconomics 15:141-155

18. Anderson RM, Fitzgerald JT, Wisdom K, Davis WK, Hiss RG (1997) A comparison of global vs disease-specific quality-of-life measures in patients with NIDDM. Diabetes Care 20:299-305

19. Jacobson AM, de Groot M, Samson JA (1994) The evaluation of two measures of quality of life in patients with type I and type II diabetes. Diabetes Care 17:267-274

20. Petterson T, Lee P, Hollis S, Young B, Newton P, Dornan T (1998) Well-being and treatment satisfaction in older people with diabetes. Diabetes Care 21:930-935

21. Wiesinger GF, Pleiner J, Quittan M et al (2001) Health related quality of life in patients with long-standing insulin dependent (type 1) diabetes mellitus: benefits of regular physical training. Wien Klin Wochenschr 113:670-675

22. Zoppini G, Carlini M, Muggeo M (2003) Self-reported exercise and quality of life in young type 1 diabetic subjects. Diabetes Nutr Metab 16:77-80

23. Riazi A, Bradley C, Barendse S, Ishii H (2006) Development of the Well-being Questionnaire short-form in Japanese: the W-BQ12. Health Qual Life Outcomes 4:40

24. Tabachnick BG, Fidell LS (2007) Using multivariate statistics. Allyn and Bacon, Needham Heights

25. Courneya KS, Segal RJ, Reid RD et al (2004) Three independent factors predicted adherence in a randomized controlled trial of resistance exercise training among prostate cancer survivors. J Clin Epidemiol 57:571-579

26. Ware JE Jr (2000) SF-36 health survey update. Spine (Phila Pa 1976) 25:3130-3139

27. Ligtenberg PC, Hoekstra JB, Bol E, Zonderland ML, Erkelens DW (1997) Effects of physical training on metabolic control in elderly type 2 diabetes mellitus patients. Clin Sci (Lond) 93:127-135

28. Ligtenberg PC, Godaert GL, Hillenaar EF, Hoekstra JB (1998) Influence of a physical training program on psychological well-being in elderly type 2 diabetes patients. Psychological well-being, physical training, and type 2 diabetes. Diabetes Care 21:2196-2197

29. Kirk AF, Higgins LA, Hughes AR et al (2001) A randomized, controlled trial to study the effect of exercise consultation on the promotion of physical activity in people with type 2 diabetes: a pilot study. Diabet Med 18:877-882

30. Toobert DJ, Glasgow RE, Strycker LA et al (2003) Biologic and quality-of-life outcomes from the Mediterranean Lifestyle Program: a randomized clinical trial. Diabetes Care 26:2288-2293

31. Martin CK, Church TS, Thompson AM, Earnest CP, Blair SN (2009) Exercise dose and quality of life: a randomized trial. Arch Intern Med 169:269-278

32. Hopman WM, Towheed T, Anastassiades T et al (2000) Canadian normative data for the SF-36 health survey. Canadian multicentre osteoporosis study research group. Can Med Assoc J 163:265-271 